\title{
A 72-year-old Patient with Dermoid Cyst
}

\author{
Esin KASAP ${ }^{1, *}$, Mine GENC ${ }^{1}$, Neslin SAHIN ${ }^{2}$, Esra Bahar GUR ${ }^{1}$, Oya Nermin SIVRIKOZ $^{3}$ \\ ${ }^{1}$ Department of Obstetrics and Gynecology, Sifa University School of Medicine, İzmir, Turkey \\ ${ }^{2}$ Department of Radiology, Sifa University School of Medicine, İzmir, Turkey \\ ${ }^{3}$ Department of Patology, Sifa University School of Medicine, İzmir, Turkey \\ *Corresponding author: dresincelik@windowslive.com
}

Received March 02, 2015; Revised March 10, 2015; Accepted March 16, 2015

\begin{abstract}
Introduction: It has been reported that the risk of malignant transformation is doubled in patients with mature cystic teratoma aged over 50 years. It is rare to encounter benign forms of mature cystic teratoma in elderly women. We aimed to report a 72-year-old woman with a benign cystic teratoma. A 72-year-old woman detected by ultrasonography to have a pelvic mass with a size of $14 \times 12 \times 10 \mathrm{~cm}$ was admitted to our department for detailed investigations. A mature cystic teratoma was diagnosed on the basis of transvaginal ultrasonography, magnetic resonance imaging, and serum concentrations of tumor markers. Postoperative pathology examination confirmed the diagnosis. This report once again showed that although mature cystic teratoma is rare in elderly women, it still occurs.
\end{abstract}

Keywords: mature cystic teratoma, postmenopausal, malignant change

Cite This Article: Esin KASAP, Mine GENC, Neslin SAHIN, Esra Bahar GUR, and Oya Nermin SIVRIKOZ, “A 72-year-old Patient with Dermoid Cyst.” American Journal of Medical Case Reports, vol. 3, no. 4 (2015): 105-107. doi: 10.12691/ajmcr-3-4-5.

\section{Introduction}

Dermoid cysts (mature cystic teratoma) constitute more than 95\% of teratomas, Mature Cystic Teratoma (MCT) constitutes $20-25 \%$ of all ovarian neoplasms, being the most common ovarian tumour of young women [1]. Women of reproductive age are affected the most; prepubertal females are rarely involved while this neoplasm is not infrequent in postmenopausal women. All three germ layers, that is ectoderm, mesoderm, and endoderm, are included by the neoplasm in a differentiated form, although parts of ectodermal origin usually make up the greatest percentage. Hence, they are alternatively called as dermoid cyst [2]. The most common elements found in dermoid cysts are of ectodermal origin such as skin, hair, hair follicles, and sebaceous or sweat glands [3]. It has been reported that the median age at diagnosis of malignant transformation of MCT was 54-61.5 years, and tended to be older than that of its benign counterpart [4]. We now report a 72year-old woman with a benign cystic teratoma.

\section{Case Report}

A 72-year-old, woman presented to urology outpatient clinic with abdominal distention, urinary incontinence, and constipation. An abdominal ultrasonography examination revealed a large cystic mass measuring $140 \times 120 \mathrm{~mm}$ at the right adnexal region containing numerous floating, highly echogenic round masses within it. She was referred to the Obstetrics and Gynecology
Department for further assessment. There was no notable medical or surgical history. Magnetic Resonance (MR) imaging, performed to determine the nature of the lesions and to evaluate the effects of this mass on adjacent structures, confirmed the sonographic findings.

On MR imaging, T1-weighted images (Figure 1A) showed a large $14 \times 12 \times 10 \mathrm{~cm}$ cystic lesion involving the pelvis, extending superiorly to the right adnexal region and displacing the uterus and urinary bladder. There were multiple round masses within the cyst. T2-weighted MR images (Figure 1B) revealed multiple round masses floating in high-signal-intensity fluid within the cyst. Fat saturated T1- and T2-weighted images demonstrated slightly diminished signal intensity within the small masses. On post-contrast T1-weighted images, there was no contrast enhancement within the lesion. Serum tumor markers were within the normal range (CA $125=10$ $\mathrm{U} / \mathrm{mL}$, CEA $=2.3 \mathrm{ng} / \mathrm{mL}$, CA $19-9=5 \mathrm{U} / \mathrm{mL})$.At laparotomy a large mass of $14 \times 12 \mathrm{~cm}$ was seen. Right salpingo-oophorectomy via laparotomy performed in November 2014 revealed a right ovarian cyst greater than $12-14 \mathrm{~cm}$ but no ascites. Intraoperative evaluation of the histology of a frozen section of the specimen showed a mature cystic teratoma; the patient was operated with total hysterectomy and bilateral adnexectomy. The patient recovered without complications, and the prognosis was favorable.

Histological findings: The ovarian material with a tumor-like appearance had an intact capsule. There were cystic areas in its cross-section. A fatty material containing hairy structures was resected from the cyst. The obtained samples were embedded into paraffin blocks after routine tissue processing protocol. Sections of 4 
micron thick were stained with hematoxylin-eosin (H\&E). Microscopic examination of the tumor demonstrated keratin areas on the inner surface lined by mature squamous epithelium below, with hair follicles and sebaceous glands underneath them (Figure 2A). Cartilage and intestinal tissue as well as serous and mucinous glands were also noted (Figure 2B). No immature tissue was seen. Based on the above findings the mass was diagnosed “mature cystic teratoma” (Figure 3).

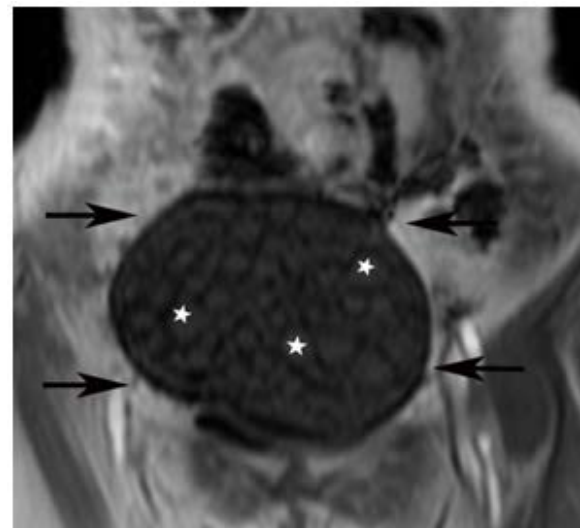

$\mathbf{A}$

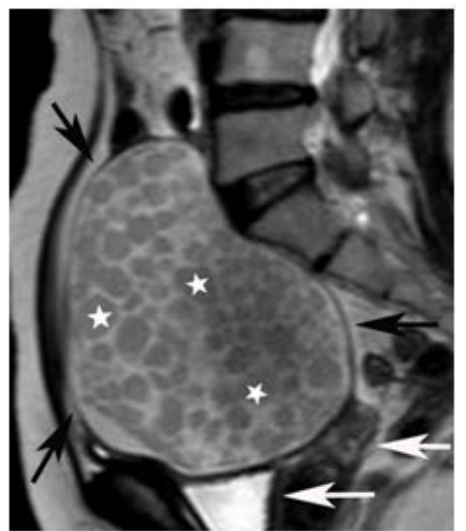

B

Figure 1. Dermoid cyst and intracystic nondepent intracystic spheres of lipid material. (a) T1-weighted coronal image shows a large cyst (black arrows) containing mutipl round masses (stars) extending süperiorly toward the right adnexal region. (b) T2-weighted sagittal MR image shows the round masses (stars) floating in high-signal-intensity fluid within the cyst (black arrow). The cystic lesion displaces the uterus and urinary bladder (white arrows)

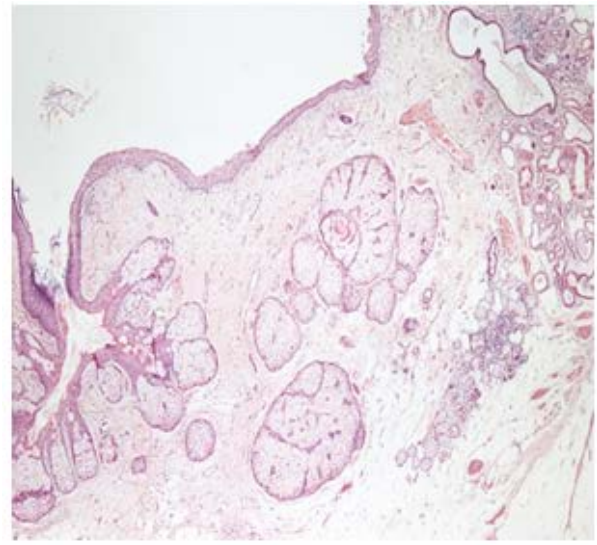

A

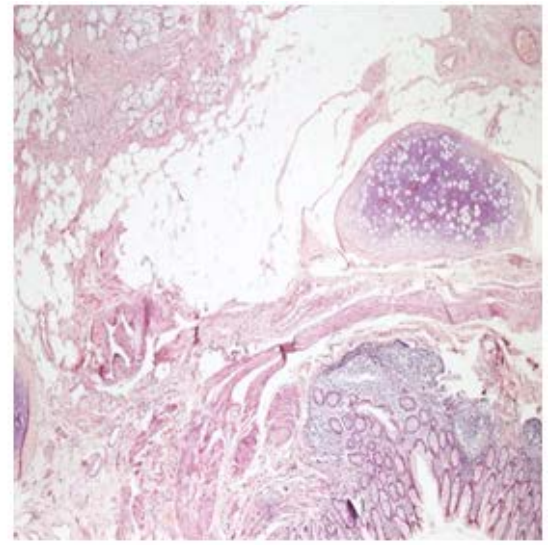

B

Figure 2 A: Areas of mature squamous epithelium, sebaceous glands, and fat tissue are observed (H\&E X40) Figure 2B: Areas of cartilage, mucous glands, and intestinal mucosa are observed (H\&E X40)

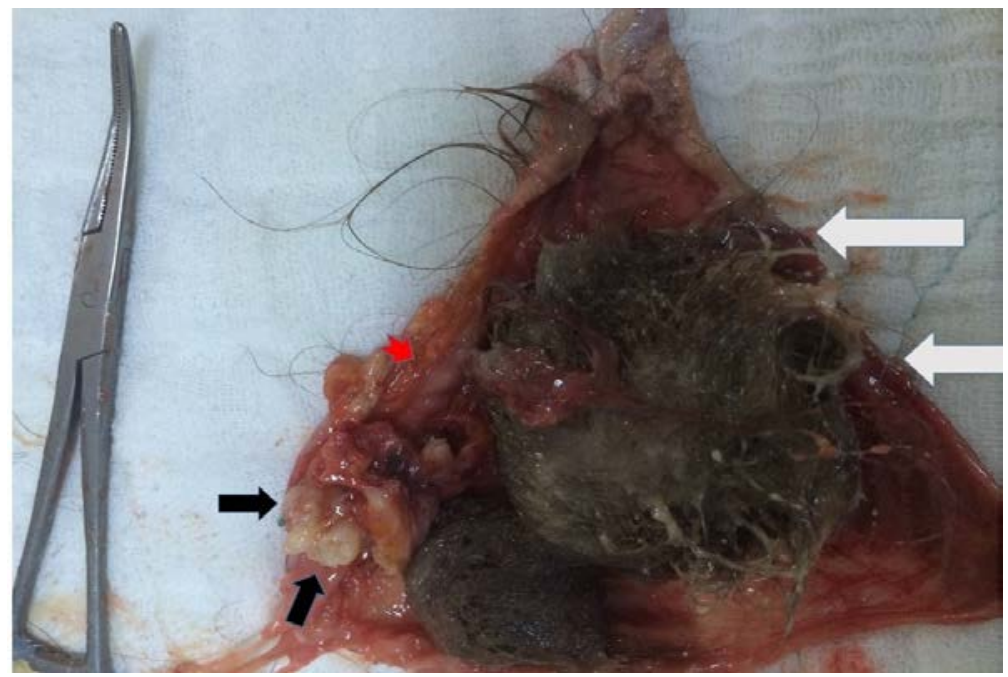

Figure 3. Photograph of the gross specimen shows yellowish, pasty sebaceous material (red arrowhead) and the bulk of the cyst cavity is filled with hair (white arrow), findings that account for the fat echogenicity and signal intensity seen at US and MR imaging. Two molar teeth are also evident ( black arrows) 


\section{Discussion}

Mature cystic teratoma is the most prevalent germ cell tumor in women of reproductive age.

Malignant transformation starts at the postmenopausal period with an average age of 55 years; the most common malignant transformation occurs to squamous cell carcinoma [5]. A unilocular lesion was shown on MR imaging. T1-weighted MR images characterized the fatty areas of the tumor as having a high signal intensity. Fatsuppressed T1-weighted images showed suppression of the signals, as well as chemical shift artifacts between the fat and the non-fat components, the latter being diagnostic for dermoid cysts. Based on a different appearance than multilocular or solid ovarian tumors, the tumor was considered benign. It has been reported that about $1 \%$ to $2 \%$ of mature cystic teratomas ultimately transform into malignant forms. This transformation usually begins from the "dermoid plug" [6]. Since the malignant transformation is rare and the malignant form resembles the appearance of the benign form, it is challenging to make a preoperative diagnosis. Malignant transformation usually occurs after menopause and when it occurs, the prognosis is grave [7]. Rim et al reported that the malignant transformation occurred at an average age of 56.8 years, and $63 \%$ of the cases were older than 40 years old at the time of diagnosis [8].

Malkasian et al reported that in a 612-case series the malignancy risk was doubled in patients over 50 years of age [9].

Yeol Park et al. [10] reported that age at the time of diagnosis and tumor size performed well than levels of CA125 or CA19-9 in detection of malignant transformation in mature cystic teratomas. They indicated that 45 years of age as the patient age and $99 \mathrm{~mm}$ as the tumor size were the optimal cutoffs. Malignant ovarian tumors may sometimes show an elevated level of SCC, the marker for squamous cell carcinoma, although tumor markers in our patient were in normal limits. It was also noteworthy that the tumor in our patient was benign despite its largest diameter of $14 \mathrm{~cm}$.

To our opinion, mature cystic teratomas are rare in the elderly women although they may well occur in some patients and this case highlights the importance of the awareness of the possibility of a benign form of the disease despite a very advanced age.

\section{References}

[1] Bal A, Mohan H, Singh SB. Malignant transformation in mature cystic teratoma of the ovary: report of five cases and review of the literature. Arch Gynecol Obstet 2007; 275: 179-82.

[2] Novak ER, Woodruff JD. Novak's Gynecologic and Obstetric Pathology With Clinical and Endocrine Relations. 8th ed. Philadelphia, PA: WB Saunders Co; 1979: 476-503.

[3] Saba L, Guerriero S, Sulcis R, ve ark. Mature and immature ovarian teratomas: CT, US and MR imaging characteristics. Eur J Radiol. 2009 Dec; 72 (3); 454-63.

[4] Dos Santos L, Mok E, Iasonos A, et al. Squamous cell carcinoma arising in mature cystic teratoma of the ovary:a case series and review of the literature. Gynecol Oncol 2007; 105: 321-4.

[5] Hackethal A, Brueggmann D, Bohlmann MK, ve ark. Squamouscell carcinoma in mature cystic teratoma of the ovary: systematic review and analysis of published data. Lancet Oncol 2008 Dec; 9 (12): 1173-80.

[6] Peterson WF, Prevost EC, Edmunds FT, Hundly JM Jr, Morris FK. Benign cystic teratomas of ovary - a clinicostatistical study of 1,007 cases with a review of the literature. Am J Obstet Gynecol. 1995; 70: 368-382.

[7] Wu RT, Torng PL, Chang DY, Chan CK, Chen RJ, Lin MC, Huang SC. Mature cystic teratoma of the ovary: a clinicopathologic study of 283 cases. Chung Hua I Hsueh Tsa Chih (Taipei). 1996; 58: 269-274.

[8] Rim SY, Kim SM, Choi HS. Malignant transformation of ovarian mature cystic teratoma. Int J Gynecol Cancer 2006; 16: 140-4.

[9] Markasian GD, Dockerty MB, Symonds RE: Benign cystic teratomas. Obstet Gynecol. 1967; 29: 719-725.

[10] Yeol Park J, Yeon Kim D, Hyeok Kim J,Man Kim Y et al.Malignant transformation of mature cystic teratoma of the ovary: Experience at a single institution. European Journal of Obstetrics \& Gynecology and Reproductive Biology. 2008; 141: 173-178. 